\title{
Avaliação da reprodutibilidade das medidas da camada de fibras nervosas retiniana e da cabeça do nervo óptico pela tomografia de coerência óptica
}

\author{
Evaluation of the reproducibility of retinal nerve fiber layer thickness \\ and optic nerve head parameters with optical coherence tomography
}

Ricardo Rau' ${ }^{1,2}$, André Luiz de Freitas Silva ${ }^{1,2}$, André Luiz Alves Salame², Luiz Alberto Soares Melo Junior ${ }^{2}$

\begin{abstract}
RESUMO
Objetivo: Determinar a reprodutibilidade da espessura da camada de fibras nervosas da retina peripapilar e dos parâmetros da cabeça do nervo óptico em olhos normais, utilizando a tomografia de coerência óptica de domínio espectral (SD-OCT).

Métodos: Trinta e quatro olhos de 34 indivíduos saudáveis foram incluídos no estudo. O protocolo do cubo do disco óptico 200x200 do OCT Cirrus foi utilizado para gerar 3 imagens de cada olho para avaliar a reprodutibilidade. O coeficiente de correlação intraclasse (ICC) foi calculado para as medidas da espessura da camada de fibras nervosas da retina e da cabeça do nervo óptico. Resultados: A correlação foi excelente para todas as medidas da espessura da camada de fibras nervosas da retina (ICC entre 0,87 e 0,98$)$. O mesmo ocorreu com os parâmetros da cabeça do nervo óptico que, com exceção da razão E/D vertical (ICC 0,56), apresentou ICC entre 0,83 e 0,99. Conclusão: As medidas de espessura da camada de fibras nervosas da retina peripapilar e os parâmetros da cabeça no nervo óptico demonstraram uma excelente reprodutibilidade com o OCT Cirrus, indicando que este aparelho poderá ser uma ferramenta útil no estudo do glaucoma. Este estudo apresenta algumas limitações, como o pequeno número de casos avaliados, sendo necessários maiores estudos para corroborar nossos achados.
\end{abstract}

Descritores: Tomografia de coerência óptica; Nervo óptico; Glaucoma; Reprodutibilidade dos testes; Retina

\begin{abstract}
Purpose: To determine the reproducibility of the peripapillary retinal nerve fiber layer thickness and optic nerve head parameters measured with the optical coherence tomography (SD-OCT) in normal eyes. Methods: Thirty-four eyes of 34 healthy patients were included in the study. The Cirrus OCT optic disc cube $200 \times 200$ protocol was used to obtain three scans from each eye to evaluate the reproducibility. Intraclass correlation coefficient (ICC) was calculated for the measurements of retinal nerve fiber layer thickness and optic nerve head parameters. Results: The correlation was excellent for all measures of retinal nerve fiber layer thickness (ICC between 0.87 and 0.98). The same happened with the optic nerve head parameters (ranging from 0.83 to 0.99), with the exception of E/D vertical ratio (0.56). Conclusion: Measurements of peripappilary retinal nerve fiber layer thickness and optic nerve head parameters showed excellent reproducibility with Cirrus OCT, indicating that this instrument may be useful in the study of glaucoma. This study has some limitations, including the small number of cases studied, further studies are needed to corroborate our findings.
\end{abstract}

Keywords: Tomography, optical coherence; Optic nerve; Glaucoma; Reproducibility of results; Retina

\footnotetext{
${ }^{1}$ Hospital Servidor Público Estadual de São Paulo "Francisco Morato de Oliveira - (HSPE-FMO) - São Paulo (SP), Brasil;

2 Escola Paulista de Medicina, Universidade de São Paulo (UNIFESP) - São Paulo (SP), Brasil.

Trabalho realizado no Hospital Servidor Público Estadual de São Paulo " Francisco Morato de Oliveira" (HSPE-FMO) - São Paulo (SP), Brasil
}

Os autores declaram inexistir conflitos de interesse

Recebido para publicação em 8/7/2011 - Aceito para publicação em 27/9/2011 


\section{INTRODUÇÃO}

$\mathbf{0}$ glaucoma é uma neuropatia óptica crônica progressiva, caracterizada por alterações típicas da cabeça do nervo óptico (CNO) e da camada de fibras nervosas da retina (CFNR), com repercussões características no campo visual. Embora algumas formas clínicas de glaucoma possuam manifestações agudas e proeminentes, a maioria dos pacientes tem uma doença crônica que se desenvolve de modo lento e não produz sintomas até que o dano no nervo óptico se instale e a perda de campo visual seja avançada ${ }^{(1)}$. Portanto, o seguimento longitudinal para rastreamento das alterações glauco-matosas é crucial $^{(2)}$.

Há evidências de que as mudanças estruturais precedem as alterações no campo visual medidas através da campimetria computadorizada ${ }^{(3)}$. Até recentemente, as mudanças estruturais glaucomatosas eram avaliadas apenas através do exame de oftalmoscopia e fotografia do fundo do olho. No entanto, pequenas alterações são difíceis de se detectar com essas técnicas e sua interpretação é totalmente subjetiva. Para se conseguir métodos mais precisos, quantitativos e reprodutíveis para detecção e seguimento de mudanças estruturais relacionadas ao glaucoma, novos métodos computadorizados de imagem de avaliação da CFNR foram desenvolvidos ${ }^{(4-6)}$.

A tomografia de coerência óptica (OCT) é uma técnica de imagem de não-contato e não-invasiva, que explora a propriedade da interferometria de baixa coerência, para obter imagens tomográficas de alta resolução( ${ }^{(3)}$ através de cortes ópticos transversais da microestrutura de tecidos biológi$\cos ^{(5,6)}$. Essa técnica vem demonstrando ser uma valiosa tecnologia para detecção e monitoramento de uma variedade de doenças oculares, como o glaucoma ${ }^{(6,7)}$.

Nos últimos anos, foram desenvolvidas novas versões de OCT que utilizam tecnologia de domínio espectral (spectral-domain), como o OCT Cirrus (Carl Zeiss Meditec, Inc). Estes aparelhos têm maior resolução axial e maior velocidade na aquisição de imagens, quando comparados aos aparelhos de OCT com tecnologia de domínio temporal (time-domain), entre eles o OCT Stratus (Carl Zeiss Meditec, Inc., Dublin, CA) ${ }^{(2)}$.

Recentemente, foi lançado pela Carl Zeiss um programa para a avaliação da CNO com o OCT Cirrus, até então inexistente para este aparelho. Este fornece a identificação automática do disco óptico e os limites de sua escavação. A análise é gerada utilizando o programa de dados para o disco óptico 200x200 e um novo algoritmo que mede com precisão a rima neurorretiniana. ${ }^{(7)}$

Métodos objetivos de quantificação anatômica da CFNR, como o OCT, podem eliminar a variabilidade inerente aos exames subjetivos e detectar o dano glaucomatoso ou sua progressão antes mesmo que ocorra o deficit funcional visual. Este aparelho fornece medidas da CFNR, com diferenças estatisticamente significantes entre olhos glaucomatosos e normais. ${ }^{(8-15)}$ Mas, antes que esta técnica possa ser usada com sucesso, a repro-dutibilidade das medidas deve ser demonstrada. Vários estudos já demonstraram a reprodutibilidade das medidas da espessura da CFNR peripapilar utilizando o OCT de domínio espectral, mas poucos estudaram a repro-dutibilidade das medidas da $\mathrm{CNO}^{(16-21)}$

O objetivo do presente estudo é determinar a reprodutibilidade da espessura da camada de fibras nervosas da retina peripapilar e dos parâmetros da cabeça do nervo óptico em olhos normais, utilizando a tomografia de coerência óptica de domínio espectral (SD-OCT).

\section{Métodos}

\section{Participantes}

Este estudo foi aprovado pelo Comitê de Ética do Hospital do Servidor Público Estadual de São Paulo, sob o número 129/10, e aderiu aos princípios da Declaração de Helsinki. O termo de consentimento livre e esclarecido foi assinado por todos os participantes.

Foram selecionados 40 indivíduos saudáveis, entre funcionários, médicos e pacientes do Serviço de Oftalmologia do Hospital do Servidor Público Estadual de São Paulo, no período de 15 de novembro a 15 de dezembro de 2010.

Os participantes foram submetidos a exame oftalmológico completo, sendo incluídos os que apresentavam acuidade visual corrigida melhor que 20/40, ausência de alterações significativas à biomicroscopia e à fundoscopia, pressão intraocular, aferida com o tonômetro de Goldmann, inferior a $21 \mathrm{mmHg}$, além de idade superior a 18 anos.

Foram excluídos os indivíduos que apresentavam disco óptico inclinado e fixação inadequada durante a realização do exame.

Apenas um olho de cada participante foi incluído no estudo, randomizado através de sorteio.

\section{Exame de OCT}

Todos os exames foram realizados pelo mesmo pesquisador, através da OCT Cirrus (software versão 5.0.0.326; Carl Zeiss Meditec, Dublin, CA, EUA), usando o protocolo do cubo do disco óptico 200x200. Em nenhum dos olhos a midríase foi realizada. O protocolo do cubo do disco óptico 200x200 destina-se a posicionar o cubo de varredura centralizado na cabeça do disco óptico.

Após o posicionamento do participante no aparelho, apoiando o queixo e a testa sobre a plataforma, a íris foi focalizada pelo examinador e a seguir a linha de varredura da imagem oftalmoscópica também foi focalizada. A CNO foi então centralizada na imagem ao vivo. Com o início do processo de digitalização da imagem, o feixe de laser de comprimento de onda de $840 \mathrm{~nm}$ gera um cubo de dados medindo 6x6mm, após a digitalização de uma série de 200 varreduras no modo B, com 200 varreduras no modo A, totalizando 40.000 pontos $^{(22)}$.

O OCT Cirrus automaticamente encontra o centro do disco óptico e extrai uma varredura no modo B na forma de um círculo de 3,46mm de diâmetro, coincidindo o centro do disco com o centro do círculo (Figura 1).

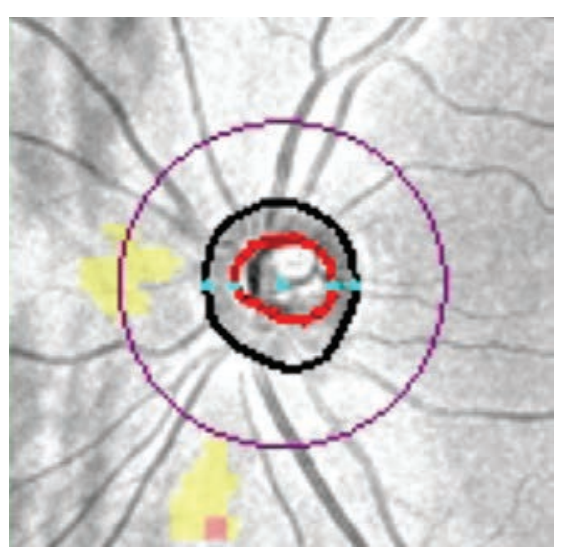

Figura 1: Círculo de 3,46 mm de diâmetro posicionado automaticamente pelo aparelho, coincidindo seu centro com o centro do disco óptico (tomografia de coerência óptica Cirrus, Carl Zeiss Meditec, Inc) 
Os limites anterior e posterior da CFNR são delineados, e a espessura é determinada em cada varredura de 200x200 no modo A. O aparelho calcula a espessura da CFNR em cada ponto sobre o círculo, sendo impressos os valores da média geral, por quadrantes e por horas (1, 2, 3, etc) (Figura 2).

Com relação às medidas da $\mathrm{CNO}$, o algoritmo identifica o término da membrana de Bruch como a borda do disco óptico. A largura da borda em toda a circunferência do disco óptico é então determinada pela medição da espessura do tecido neurorretiniano no nervo óptico, que se transforma para sair através da abertura na membrana de Bruch. Os seguintes parâmetros da CNO são analisados: área do disco, área da rima, razão escavação/disco (E/D) média, razão E/D vertical e volume da escavação ${ }^{(22)}$ (Figura 3).

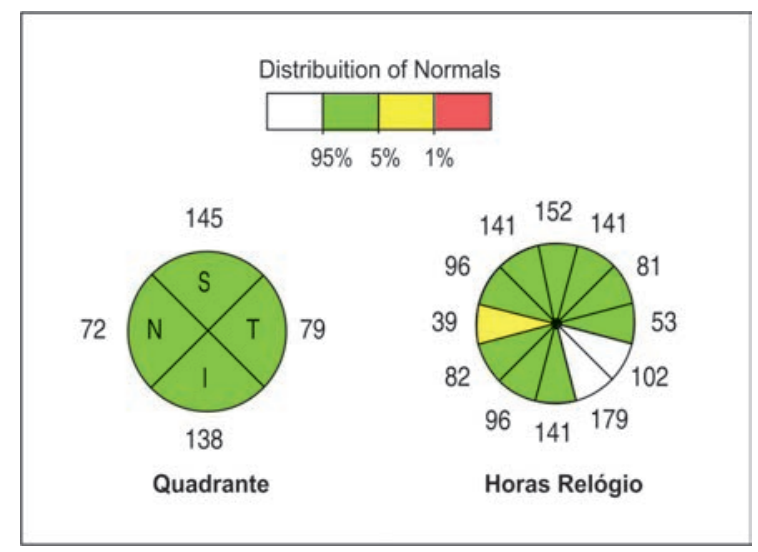

Figura 2: Medida da espessura da camada de fibras nervosas peripapilar $(\mu \mathrm{m})$, distribuída em quadrantes e horas do relógio (tomografia de coerência óptica Cirrus, Carl Zeiss Meditec, Inc)
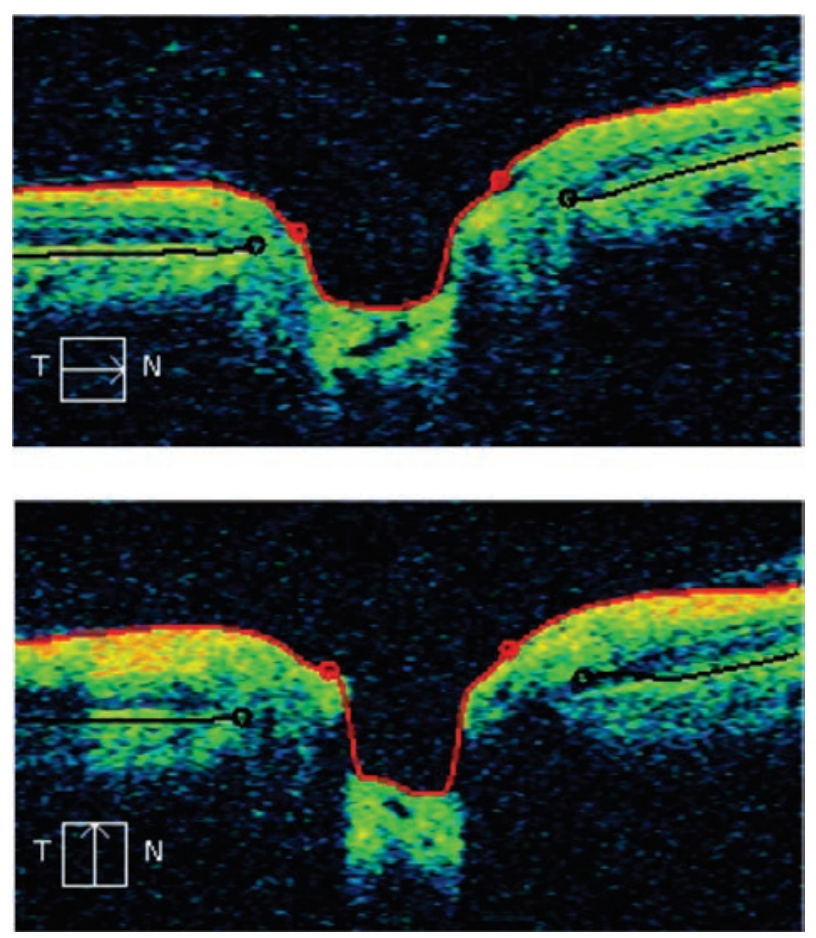

Figura 3: Tomografia horizontal e vertical para avaliação dos parâmetros da cabeça do nervo óptico (tomografia de coerência óptica Cirrus, Carl Zeiss Meditec, Inc)
Todos os parâmetros da CFNR e da CNO são gerados automaticamente por um algoritmo de análise da Carl Zeiss recentemente desenvolvido para o OCT Cirrus (software versão 5.0.0.326), que não envolve a interação do examinador.

Três imagens foram adquiridas de cada olho, entre 5-10 segundos de intervalo, para avaliação da reprodutibilidade.

Apenas os exames que apresentaram sinal de varredura $>$ 6, ausência de movimento do olho ou ausência de artefatos dentro dos $1,73 \mathrm{~mm}$ de raio ao redor da cabeça do nervo óptico foram considerados para análise.

\section{Análise estatística}

A reprodutibilidade foi avaliada pelo cálculo do coeficiente de correlação intraclasse (ICC). Um valor de ICC alto indica pequenas flutuações entre as medidas repetidas sobre os mesmos indivíduos, indicando uma alta reprodutibilidade. Já um ICC baixo indica grandes flutuações, caracterizando uma baixa reprodutibilidade. O valor máximo do ICC é 1,0, enquanto o seu valor mínimo é, teoricamente, zero. Valores ${ }^{3}$ 0,75 são considerados excelentes, entre e" 0,4 e $^{3} 0,75$ satisfatórios e ${ }^{3} 0,4$ pobres.

\section{Resultados}

Quarenta participantes foram selecionados pelos critérios de inclusão, dos quais 6 foram excluídos devido à má qualidade da imagem gerada nos exames, sendo 4 por opacidade de meios e 2 por falta de colaboração (como movimentação dos olhos e piscar excessivo). Portanto, a análise deste estudo foi baseada nos dados de 34 participantes, com características descritas na Tabela 1.

As médias dos valores da espessura da CFNR e da CNO estão dispostas na Tabela 2 .

O ICC foi excelente para todas as medidas da CFNR, com o quadrante inferior mostrando o menor valor $(0,87)$; e a média total e o quadrante temporal os maiores $(0,97$ e 0,98 , respectivamente). O mesmo ocorreu com os parâmetros da $\mathrm{CNO}$, exceto a razão E/D vertical, com valor de 0,56 (Tabela 3).

Tabela 1

Características dos pacientes avaliados

\section{Características dos participantes}

$\begin{array}{llr}\text { Sexo } & \text { Feminino } & \mathrm{N}=29 \\ \text { Masculino } & \mathrm{N}=05 \\ \text { Idade (anos) } & \text { Média } & 35,5 \pm 11,2 \\ & \text { Mínima } & 21 \\ & \text { Máxima } & 65 \\ \text { Raça } & \text { Branca } & 30 \\ & \text { Negra } & 3 \\ & \text { Amarela } & 1 \\ \text { Olho avaliado } & \text { Direito } & \mathrm{N}=15 \\ & \text { Esquerdo } & \mathrm{N}=19\end{array}$




\section{Tabela 2}

Medidas da espessura da camada de fibras nervosas da retina e da cabeça do nervo óptico

\begin{tabular}{|c|c|c|c|c|}
\hline Parâmetro & Média & Desvio-padrão & Mínimo & Máximo \\
\hline \multicolumn{5}{|l|}{$\begin{array}{l}\text { CFNR }(\mu \mathrm{m}) \\
\end{array}$} \\
\hline Média & 94,6 & 7,2 & 83,3 & 110,7 \\
\hline Quadrante superior & 119,0 & 14,4 & 95,3 & 149,3 \\
\hline Quadrante inferior & 127,1 & 13,1 & 100,7 & 153,7 \\
\hline Quadrante nasal & 67,9 & 8,1 & 53,7 & 85,0 \\
\hline Quadrante temporal & 64,2 & 11,2 & 44,0 & 91,0 \\
\hline \multicolumn{5}{|l|}{ Cabeça do nervo óptico } \\
\hline Área da rima $\left(\mathrm{mm}^{2}\right)$ & 1,38 & 0,23 & 0,88 & 1,80 \\
\hline Área do disco $\left(\mathrm{mm}^{2}\right)$ & 1,83 & 0,34 & 1,40 & 3,07 \\
\hline Razão escavação/disco média & 0,44 & 0,16 & 0,07 & 0,77 \\
\hline Razão escavação/disco vertical & 0,40 & 0,16 & 0,06 & 0,76 \\
\hline Volume da escavação $\left(\mathrm{mm}^{3}\right)$ & 0,12 & 0,16 & 0,00 & 0,91 \\
\hline
\end{tabular}

CFNR = camada de fibras nervosas da retina

Tabela 3

\section{Coeficiente de correlação intraclasse das medidas da camada de fibras nervosas da retina e da cabeça do nervo óptico}

\begin{tabular}{lr}
\hline \multicolumn{1}{c}{ Parâmetro } & ICC \\
\hline CFNR & \\
Média & 0,97 \\
Quadrante superior & 0,90 \\
Quadrante inferior & 0,87 \\
Quadrante nasal & 0,90 \\
Quadrante temporal & 0,98 \\
Cabeça do nervo óptico & \\
Área da rima & 0,85 \\
Área do disco & 0,83 \\
Razão escavação/disco média & 0,97 \\
Razão escavação/disco vertical & 0,56 \\
Volume da escavação & 0,99 \\
\hline ICC = coeficiente de correlação intraclasse; CFNR = camada de fibras nervosas da retina.
\end{tabular}

\section{DiscussÃo}

Este estudo mostrou excelente reprodutibilidade para todas as medidas de espessura da CFNR (variando de 0,87 a 0,98 ). Garas et al., (16) utilizando o RTVue-100 OCT (Optovue, Inc., Fremont, CA) também encontrou alta reprodutibilidade (entre 0,89 e 0,99 ) em todas as medidas da CFNR, nos 77 olhos estudados. Budenz et al. ${ }^{(23)}$ avaliaram 51 pacientes utilizando OCT Stratus, encontrando reprodutibilidade acima de $0,90 \mathrm{em}$ todas as medidas de espessura da CFNR, exceto no quadrante nasal $(0,67)$. Paunescu et al. ${ }^{(6)}$ relataram reprodutibilidade de 0,71 e 0,60 para os quadrantes inferior e nasal, respectivamente. $\mathrm{O}$ estudo de Kim et al. ${ }^{(18)}$ sobre a variabilidade das medidas de espessura da CFNR com o OCT Cirrus, com 14 olhos normais, encontrou um ICC de 0,984 para a espessura média da CFNR e ICC $<0,90$ em metade das medidas por quadrantes.

No geral, nos diversos estudos com indivíduos portadores de glaucoma, observou-se que a média de espessura da CFNR apresentou um dos maiores ICC. Do ponto de vista prático, isso sugere que a espessura média da CFNR, ao invés da espessura nos quadrantes pode ser o melhor parâmetro de avaliação da CFNR para o monitoramento do glaucoma ${ }^{(22)}$.

Neste estudo encontrou-se uma excelente reprodutibilidade nos parâmetros da $\mathrm{CNO}$, exceto na razão E/D vertical $(0,56)$, utilizando o OCT Cirrus. González-Garcia et al. ${ }^{(17)}$ também relataram uma ótima repro-dutibilidade para a área do disco, volume do disco e razão E/D média. Paunescu et al. ${ }^{(6)}$ examinaram 10 jovens saudáveis e observaram que a razão $\mathrm{E} / \mathrm{D}$ média e a razão E/D vertical apresentaram os melhores ICC $\left(0,97\right.$ e 0,90 , respectivamente), e a área do disco pior $\operatorname{ICC}(0,52)^{(6)}$. Olmedo et al. ${ }^{(5)}$ avaliaram a reprodutibilidade dos parâmetros da CNO em 10 olhos normais e 10 olhos glaucomatosos, encontrando nos olhos normais ICC maior que 0,81 em todos os parâmetros, exceto a área do disco $(0,74)$ e a área da rima $(0,33)$ que tiveram reprodutibilidade relativamente pobre. O ICC para os olhos glaucomatosos variou de 0,85 a 0,95 , exceto a área do disco $(0,68)$. Lin et al. ${ }^{(4)}$ também encontraram os parâmetros da CNO altamente reprodutíveis, com ICC variando entre 0,86 e 0,959 , com exceção da área do disco $(0,73)$.

Deve-se notar que, ao contrário dos estudos com o OCT Stratus, que mostram baixa reprodutibilidade das medidas da área do disco e resultados variáveis em relação aos outros parâmetros, os estudos de González-Garcia et al., ${ }^{(17)}$ utilizando OCT de domínio espectral (RTVue; Optovue, Inc. ), de Mwanza et al. ${ }^{(22)}$ e o presente estudo (utilizando o OCT Cirrus) constataram que a medida da área do disco é tão reprodutível como os demais parâmetros da $\mathrm{CNO}$ e da CFNR.

A área do disco óptico é fator de risco fraco para o glaucoma e não é influenciada pelo nível de pressão intraocular ou pela extensão do dano glaucomatoso. Portanto, isso não interfere na progressão do glaucoma ${ }^{(22)}$. No entanto, todos os outros parâmetros da CNO são diretamente dependentes da área do disco, sendo essencial que as medidas de área do disco sejam altamente reprodutíveis $^{(22)}$.

Esta variação na área do disco pode ocorrer devido à incorreta delimitação de sua margem, conforme já foi relatado anteriormente em trabalhos utilizando o algoritmo para análise da CNO com o OCT Stratus, que utiliza um plano de referência padrão, localizado $150 \mu \mathrm{m}$ acima do epitélio pigmentar da retina para definir a margem do disco ${ }^{(24)}$. Isso não ocorre no programa de análise da cabeça do nervo óptico do OCT Cirrus, que realiza as medições diretamente do disco e da escavação, dependendo apenas da anatomia, ao invés de um plano de referência ${ }^{(25)}$.

Este estudo apresenta algumas limitações. A média de idade dos participantes corresponde à faixa etária de adultos jo- 
vens e somente exames com boa qualidade de imagem (sinal de varredura $>6$ ) foram utilizados, fatores estes que podem ter levado as medidas de reprodutibilidade ao limite superior. É sabido que a população mais acometida pelo glaucoma tem idade superior a 40 anos, muitas vezes apresentam opacidade de meios (catarata, opacidades corneanas ou vítreas) e, em alguns casos, colaboram pouco durante a realização do exame. Estes fatores podem levar a um exame de qualidade ruim, que deverá ser avaliado cuidadosamente ou, muitas vezes, descartado, devendo ser considerados outros métodos para avaliação. Neste trabalho foram analisados olhos normais e, embora trabalhos com olhos glaucomatosos não tenham demonstrado diferenças na reprodutibilidade quando comparados aos normais, mais estudos com pacientes com glaucoma devem ser realizados, para se firmar a utilização do OCT no acompanhamento do glaucoma.

\section{Conclusão}

As medidas de espessura da CFNR peripapilar e os parâmetros da CNO demonstraram uma excelente reprodutibilidade com o OCT Cirrus, para este grupo de participantes avaliados, indicando que este aparelho poderá ser uma ferramenta útil no estudo do glaucoma. Entretanto, estudos com um número maior de participantes, de diferentes raças e idades, são necessários para corroborar nossos achados.

\section{REFERÊNCIAS}

1 Sung KR, Kim DY, Park SB, Kook MS. Comparison of retinal nerve fiber layer thickness measured by Cirrus HD and Stratus optical coherence tomography. Ophthalmology. 2009;116(7):126470, 1270.e1.

2 Huang D, Swanson EA, Lin CP, Schuman JS, Stinson WG, Chang W, et al. Optical coherence tomography. Science. 1991; 254(5035):1178-81.

3 Kerrigan-Baumrind LA, Quigley HA, Pease ME, Kerrigan DF, Mitchell RS. Number of ganglion cells in glaucomatous eyes compared with threshold visual field test in the same persons. Invest Ophthalmol Vis Sci. 2000;41(3):741-8.

4 Lin D, Leung CK, Weinreb RN, Cheung CY, Li H, Lam DS. Longitudinal evaluation of optic disc measurement variability with optical coherence tomography and confocal scanning laser ophthalmoscopy. J Glaucoma. 2009;18(2):101-6.

5 Olmedo M, Cadarso-Suarez C, Gomez-Ulla F, Val C, Fernandez I. Reproducibility of optic nerve head measurements obtained by optical coherence tomography. Eur J Ophthalmol. 2005;15(4):486-92.

6 Paunescu LA, Schuman JS, Price LL, Stark PC, Beaton S, Ishikawa $\mathrm{H}$, et al. Reproducibility of nerve fiber thickness, macular thickness, and optic nerve head measurements using StratusOCT. Invest Ophthalmol Vis Sci. 2004;45(6):1716-24.

7 Cirrus HD-OCT Software Version 5: A new level of clinical certainty. Available from: www.zeiss.com

8 Schuman JS, Pedut-Kloizman T, Hertzmark E, Hee MR, Wilkins JR, Coker JG, et al. Reproducibility of nerve fiber layer thickness measurements using optical coherence tomography. Ophthalmology. 1996;103(11):1889-98. Comment in Ophthalmology. 1997;104 (10):1530-1.

9 Zangwill LM, Bowd C, Berry CC, Williams J, Blumenthal EZ, Sánchez-Galeana CA, et al. Discriminating between normal and glaucomatous eyes using the Heidelberg Retina Tomograph, GDx Nerve Fiber Analyzer, and Optical Coherence Tomograph. Arch Ophthalmol. 2001;119(7):985-93.

10 Bowd C, Zangwill LM, Berry CC, Blumenthal EZ, Vasile C, Sanchez-Galeana C, et al. Detecting early glaucoma by assessment of retinal nerve fiber layer thickness and visual function. Invest Ophthalmol Vis Sci. 2001;42(9):1993-2003.
11 Mok KH, Lee VW, So KF. Retinal nerve fiber loss pattern in high-tension glaucoma by optical coherence tomography. J Glaucoma. 2003;12(3):255-9.

12 Ophir A. Optical coherence tomography measurement of nerve fiber layer thickness and the likelihood of a visual field defect. Am J Ophthalmol. 2003;135(5):744; author reply 744-5. Comment on Am J Ophthalmol. 2002;134(4):538-46.

13 Kee C, Cho C. Evaluation of retinal nerve fiber layer thickness in the area of apparently normal hemifield in glaucomatous eyes with optical coherence tomography. J Glaucoma. 2003;12(3):250-4.

14 Varma R, Bazzaz S, Lai M. Optical tomography-measured retinal nerve fiber layer thickness in normal latinos. Invest Ophthalmol Vis Sci. 2003;44(8):3369-73.

15 Lachkar Y. [Automated imaging of the optic nerve and optic nerve fibers is essential to daily clinical practice]. J Fr Ophtalmol. 2004;27(6 Pt 2):724-9. French.

16 Garas A, Tóth M, Vargha P, Holló G. Comparison of repeatability of retinal nerve fiber layer thickness measurement made using the RTVue Fourier-domain optical coherence tomograph and the GDx scanning laser polarimeter with variable or enhanced corneal compensation. J Glaucoma. 2010;19(6):412-7.

17 González-García AO, Vizzeri G, Bowd C, Medeiros FA, Zangwill LM, Weinreb RN. Reproducibility of RTVue retinal nerve fiber layer thickness and optic disc measurements and agreement with Stratus optical coherence tomography measurements. Am J Ophthalmol. 2009;147(6):1067-74, 1074.e1.

18 Kim, JS, Ishikawa H, Sung KR, Xu J, Wollstein G, Bilonick RA, et al. Retinal nerve fiber layer thickness measurement reproducibility improved with spectral domain optical coherence tomography. Br J Ophthalmol. 2009;93(8):1057-63.

19 Leung CK, Cheung CY, Weinreb RN, Qiu Q, Liu S, Li H, et al. Retinal nerve fiber layer imaging with spectral-domain optical coherence tomography: a variability and diagnostic performance study. Ophthalmology.2009;116(7):1257-63.

20 Menke MN, Dabov S, Knecht P, Sturm V. Reproducibility of retinal thickness measurements in healthy subjects using spectralis optical coherence tomography. Am J Ophthalmol. 2009;147(3):467-72.

21 Vizzeri G, Weinreb RN, Gonzalez-Garcia AO, Bowd C, Medeiros FA, Sample PA, Zangwill LM. Agreement between spectral-domain and time-domain OCT for measuring RNFL thickness. Br J Ophthalmol. 2009;93(6):775-81.

22 Mwanza JC, Chang RT, Budenz DL, Durbin MK, Gendy MG, Shi W, Feuer WJ. Reproducibility of peripapillary retinal nerve fiber layer thickness and optic nerve head parameters measured with cirrus HD-OCT in glaucomatous eyes. Invest Ophthalmol Vis Sci. 2010;51(11):5724-30.

23 Budenz DL, Fredette MJ, Feuer WJ, Anderson DR. Reproducibility of peripapillary retinal nerve fiber thickness measurements with stratus OCT in glaucomatous eyes. Ophthalmology 2008;115(4):661-666.e4.

24 Marsh BC, Cantor LB, WuDunn D, Hoop J, Lipyanik J, Patella $\mathrm{VM}$, et al. Optic nerve head (ONH) topographic analysis by stratus OCT in normal subjects: correlation to disc size, age, and ethnicity. J Glaucoma. 2010;19(5):310-8.

25 Ortega Jde L, Kakati B, Girkin CA. Artifacts on the optic nerve head analysis of the optical coherence tomography in glaucomatous and nonglaucomatous eyes. J Glaucoma. 2009;18(3):186-91.

\section{Autor correspondente:}

Ricardo Rau

Rua Bartolomeu de Gusmão, $n^{\circ}$ 430, apto 91

Vila Mariana - CEP 04111-021 - São Paulo (SP), Brasil

E-mail:ricardorau@gmail.com 